\title{
Expression of the Soluble Extracellular Domain of Human Thrombopoietin Receptor Using a Maltose-Binding Protein-Affinity Fusion System
}

\author{
Qing Zhang, Rui-Min Pan, Yi-Chen Ge, and Peilin Xu* \\ Biotechnology Research Center, Key Laboratory of Gene Engineering of the Education Ministry, Zhongshan University; \\ Guangzhou 510275, The People's Republic of China. Received September 2, 2003; accepted October 20, 2003
}

\begin{abstract}
The thrombopoietin (TPO) receptor (Mpl) belongs to the family of ligand-dependent cytokine receptors and plays a functional role in regulating platelet production. The signaling capacity largely depends on the binding of TPO to the extracellular domains of the TPO receptor (Mpl-EC). Because the expression level of Mpl in human tissue is very low, studies on the functional and spatial characteristics of its ligand-binding sites have been limited. In the present study, we report the expression and purification of Mpl-EC as a fusion with the maltose-binding protein (MBP), designated MBP-Mpl-EC. MBP-Mpl-EC was expressed in the cytoplasm of Escherichia coli as a soluble fusion protein. Specific binding of TPO to purified MBP-Mpl-EC was demonstrated by a dot-blot assay and surface plasmon resonance. We conclude that bacterial expression of MBP-Mpl-EC yields large amounts of protein with correct folding and that it can be used for further structure and function analyses.
\end{abstract}

Key words thrombopoietin; $\mathrm{cMpl}$; platelet; maltose-binding protein; expression; fusion protein

The binding of thrombopoietin (TPO) to its cognate receptor, Mpl, triggers a series of intracellular signal transductions to stimulate mature megakaryocytes that respond by increasing platelet production. TPO cDNA was cloned in 1994, and the product of TPO was described as consisting of two domains, a $N$-terminal cytokine domain (150 amino acids) and a larger $C$-terminal domain (203 amino acids). ${ }^{1,2)}$ The $N$-terminal TPO is responsible for binding to its receptor, leading to dimerization in which two receptor molecules bind one ligand at the extracellular domain of $\mathrm{Mpl}(\mathrm{Mpl}-\mathrm{EC}){ }^{3,4)} \mathrm{Mpl}$ belongs to the cytokine receptor superfamily. Two types of human Mpl, MPLP and MPLK, were previously identified. Both types of $\mathrm{Mpl}$ contain extracellular domains of 485 amino acids with a leader sequence of 18 amino acids and a transmembrane domain of 22 amino acids, but differ in their cytoplasmic domains. ${ }^{5)}$ Mpl-EC contains two cytokine subunits that display a distinctive conservation of four cysteine residues at their $N$-termini and the consensus sequence WSXWS at the $C$-termini. $\left.{ }^{6}\right)$ Previous investigations of the binding between TPO and Mpl were hampered by the difficulty in producing $\mathrm{Mpl}$ in large quantities. In this paper, we report the expression and purification of human Mpl-EC coupled to and expressed as a fusion product with maltose-binding protein (MBP), in Escherichia coli. The fusion protein was demonstrated to be biochemically active in specifically binding with its ligand TPO.

\section{MATERIALS AND METHODS}

Materials A pMal protein expression and purification system, including pMal-c2X, MBP antibody, and amylose resin, were purchased from New England Biolabs (Beverly, MA, U.S.A.). Bacterial strain BL21(DE3)pLysS was purchased from Novagen (Madison, WI, U.S.A.). Recombinant mouse TPO was obtained from Sigma Chemical (St. Louis, MO, U.S.A.).

Construction of the Expression Plasmid pMal-Mpl-EC The DNA fragment encoding Mpl-EC (26-491) without the signal peptide was amplified by PCR using previously con- structed plasmid pET-TPOR-EN ${ }^{7)}$ as a template, and the sense primer 5'-CCGGAA TTCATGAGC CAAGATGTCTCCTTGCTG-3' and antisense primer 5'-ATA GTCGACTTA AGTTGGGTCCGACCACGAGCT-3'. EcoRI and SalI (underlined) were introduced into the sense and antisense primers, respectively. The PCR product was subcloned into EcoRI-and SalI-digested expression vector pMal-c2X. The construct was confirmed by nucleotide sequencing and was used to transform E. coli strain BL21(DE3)pLysS.

Expression and Purification of MBP-Mpl-EC E. coli strain BL21 (DE3) expressing MBP-Mpl-EC was grown in LB medium containing ampicillin $0.1 \mathrm{mg} / \mathrm{ml}$ to an $\mathrm{A}_{600}$ of 0.7 at $37^{\circ} \mathrm{C}$. The expression was induced with $0.5 \mathrm{~mm}$ IPTG for $3 \mathrm{~h}$ at $30^{\circ} \mathrm{C}$. Cells were collected by centrifugation and resuspended in a binding buffer containing $20 \mathrm{~mm}$ Tris, $\mathrm{pH}$ 7.4, $150 \mathrm{~mm} \mathrm{NaCl}, 0.1 \mathrm{~mm}$ PMSF, $1 \mathrm{~mm}$ EDTA, and $0.5 \mathrm{~mm}$ dithiothreitol, and frozen at $-80^{\circ} \mathrm{C}$. The bacteria was thawed at $37^{\circ} \mathrm{C}$ and lysed by sonication. The soluble faction containing MBP-Mpl-EC fusion protein was loaded on a prepacked amylose affinity column, washed with 3 column volumes with a buffer of $20 \mathrm{~mm}$ Tris, $\mathrm{pH} 7.4$, and $150 \mathrm{~mm} \mathrm{NaCl}$. Elution was performed with the same buffer supplemented with $10 \mathrm{~mm}$ maltose.

Electrophoresis and Western-Blot Analysis Protein was analyzed by sodium dodecyl sulfate-polyacrylamide gel electrophoresis (SDS-PAGE) on 10\% polyacrylamide gel. After electrophoresis, the gel was soaked in transfer buffer ( $48 \mathrm{~mm}$ Tris, $39 \mathrm{~mm}$ glycine, $0.037 \% \mathrm{w} / \mathrm{v}$ SDS in $20 \% \mathrm{v} / \mathrm{v}$ methanol). Protein was transferred to PVDF membrane using a Hoefer SemiPhor semi-dry transfer unit (Pharmacia, Uppsala, Sweden). The PVDF membrane was blocked by adding $1 \%$ bovine serum albumin (BSA) in Tris-buffered Tween-20 (TBT) overnight at $4{ }^{\circ} \mathrm{C}$, followed by incubation with rabbit polyclonal serum raised against MBP diluted in blocking solution. Immunoreactive materials were detected using alkaline phosphatase-conjugated goat anti-rabbit antibodies (MBI Fementas, $1: 10000$ ).

Dot-Blot Assay Binding interaction between TPO and purified MBP-Mpl-EC was performed using a dot blot analy- 
sis. TPO was diluted in PBS buffer, $\mathrm{pH}$ 7.4, at concentration ranging from $12.5 \mathrm{ng} / \mu \mathrm{l}$ to $200 \mathrm{ng} / \mu \mathrm{l}, 5 \mu \mathrm{l}$ each sample was spotted onto a PVDF membrane using a dot-blot filtration apparatus, and the membrane was blocked with $1 \% \mathrm{BSA}$ in TBT buffer for $1 \mathrm{~h}$. The membrane was then incubated with purified MBP-Mpl-EC $(0.5 \mu \mathrm{g} / \mathrm{ml})$ in blocking buffer overnight at $4{ }^{\circ} \mathrm{C}$. After washing with TBT buffer, the membrane was incubated with anti-MBP polyclonal antibody for $2 \mathrm{~h}$ at room temperature. After extensively washing the membrane again with TBT buffer, bound protein was detected using alkaline phosphatase-conjugated goat anti-rabbit antibodies.

Surface Plasmon Resonance Analysis The procedures were essentially in accordance with the Affinity Sensor instruction manual (Affinity Sensors, Cambridge, U.K.). Briefly, the cuvette was equilibrated at $25^{\circ} \mathrm{C}$ in PBS/T $(10 \mathrm{~mm}$ phosphate, $2.7 \mathrm{~mm}$ potassium chloride, $137 \mathrm{~mm}$ sodium chloride, $0.05 \%$ Tween 20 ) for $10 \mathrm{~min}$, followed by activation for $7 \mathrm{~min}$ with a freshly prepared NHS/EDC mixture. MBP-Mpl-EC was added and left for $10 \mathrm{~min}$. Uncoupled MBP-Mpl-EC was washed out with PBS/T. Any nonreacted carboxyl groups were deactivated with $1 \mathrm{M}$ ethanolamine, $\mathrm{pH}$ 8.5. Binding assays were performed in $100 \mu \mathrm{l}$ of PBS (120 mM NaCl, $2.7 \mathrm{~mm} \mathrm{KCl}, 10 \mathrm{~mm}$ phosphate, $\mathrm{pH}$ 7.4). Dissociation was performed by replacing the TPO with $100 \mu \mathrm{l}$ of PBS. The cuvette was regenerated by washing with $20 \mathrm{~mm} \mathrm{HCl}$ for $1 \mathrm{~min}$. Both $k_{\text {ass }}$ and $k_{\text {diss }}$ rate constants were calculated according to the first-order equation: $R_{t}=R_{\mathrm{o}}+$ $E\left[1-\exp \left(-k_{\mathrm{on}} t\right)\right]$ where $R_{t}$ is the response at time $t ; R_{\mathrm{o}}$ is the initial response; $E$ is the extent of the response and $k_{\text {on }}$ is the on-rate constant.

\section{RESULTS AND DISCUSSION}

Construction and Expression of MBP-Mpl-EC Fusion Protein The cDNA encoding Mpl-EC lacking it signal peptide was cloned into the pMal-c2X vector, downstream and in frame with the malE gene encoding MBP to give the construct pMal-Mpl-EC (Fig. 1). After sequencing the clone insert, it showed an identical sequence to that previously described for $\mathrm{Mpl}$ cDNA (GenBank Accession No. NM 005373). A construct was used to transform E. coli BL21(DE3)pLysS as a bacterial expression host. Different concentrations of IPTG $(0.5,1,2,5 \mathrm{mM})$ were compared for inducing the expression of protein, and induction with $0.5 \mathrm{~mm}$ IPTG yielded a higher level of expression (data not shown). In the cellular lysate of E. coli, the band of $90 \mathrm{kDa}$, corresponding to MBP-Mpl-EC, was clearly shown on SDSPAGE after Coomassie blue staining (Fig. 2A). The bacterial lysate was subjected to centrifugation to analyze the solubility of the MBP-Mpl-EC fusion protein. About $50 \%$ of the protein produced with pMal-Mpl-EC was present in the soluble fraction, whereas almost half appeared to be insoluble (data not shown). In an attempt to minimize the formation of inclusion bodies and increase the yield of the soluble fraction, the growth conditions of the bacteria were altered so that the temperature of the incubation was decreased from 30 to $25^{\circ} \mathrm{C}$. However, the expression of soluble protein did not increase with incubation at the lower temperature (data not shown).

Purification of MBP-Mpl-EC Fusion Protein The sol- uble form of MBP-Mpl-EC from the crude extract fraction was directly purified using an amylose-agarose column. After elution of the fusion protein from the column, the quantity of the purified protein was determined using the Bradford assay (Bio-Rad) with BSA as a standard. The total amount of protein recovered was estimated to be $10 \mathrm{mg}$ per liter of bacterial culture. The purified protein was run on $10 \%$ polyacrylamide gel along with the samples of lysate and crude extract. Figure 2 shows a band at $90 \mathrm{kDa}$, which is the expected size of the fusion protein. The purity of MBP-Mpl-EC was estimated to be greater than $85 \%$ (Fig. 2A). A band of the expected size was detected in Western blotting by anti-MBP antibodies directed against the fusion protein (Fig. 2B).

Binding of TPO to Recombinant MBP-Mpl-EC To evaluate whether the recombinant MBP-Mpl-EC retained its biochemical function, the interaction between TPO and MBP-Mpl-EC was verified in vitro using a dot-blot assay. An equal volume of two-fold serially diluted of TPO was spotted

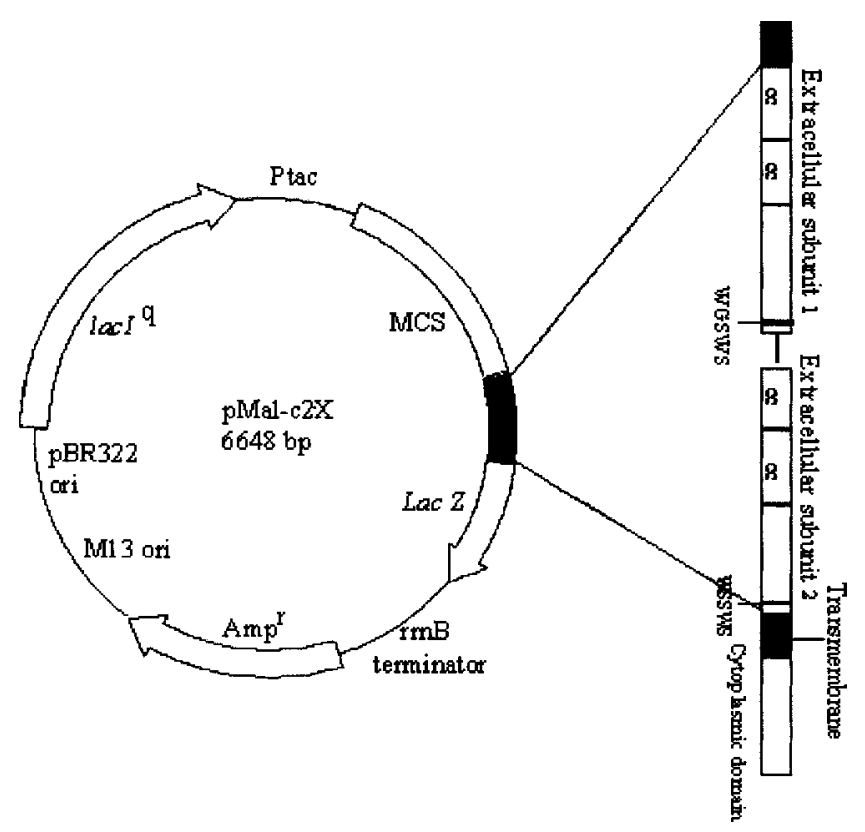

Fig. 1. Construction of the Expression Plasmid pMal-Mpl-EC

The extracellular domain of $\mathrm{Mpl}$ was inserted downstream from the MalE gene. The important regulatory elements and encoded genes are indicated.



Fig. 2. SDS-PAGE and Western-Blot Analyses of Mal-Mpl-EC

(A) Aliquots $(5 \mu \mathrm{leach})$ of the fusion protein samples were analyzed on $10 \%$ polyacrylamide gel. The gel was stained with Coomassie brilliant blue. Arrow indicates MBP-Mpl-EC. (B) The fusion protein samples were detected by immunoblotting with anti-MBP monoclone antibody. M, molecular size markers; lanes 1 and 2, bacterial cells harboring plasmid pMal-Mpl-EC induced without or with IPTG; lane 3, soluble cellular extract; 4, purified Mpl-EC after amylose-affinity chromatography. 
onto a PVDF membrane and incubated with the purified MBP-Mpl-EC or MBP, and binding was monitored by treating the dot-blot with the MBP-specific antibody. Quantification results are presented in Fig. 3, indicating that the fusion protein had saturable high-affinity binding for TPO, whereas no nonspecific binding occurred in the control experiment with MBP.

We next determined the rate constants of binding association $\left(k_{\text {ass }}\right)$ and dissociation $\left(k_{\text {diss }}\right)$ between MBP-Mpl-EC and TPO using the IAsys Affinity Biosensor. The purified MBPMpl-EC protein was immobilized covalently to the surface of a carboxymethyl dextran cuvette and hybridized with TPO at varied concentrations, ranging from $0.41 \mu \mathrm{M}$ to $2.06 \mu \mathrm{M}$. Figure 4 depicts the specific interaction between TPO and immobilized MBP-Mpl-EC. The $k_{\text {ass }}$ and $k_{\text {diss }}$ values were $7572.45 \mathrm{~m}^{-1} \mathrm{~s}^{-1}$ and $0.00389 \mathrm{~m}^{-1}$, respectively, calculated using the FASTfit program algorithm. ${ }^{8,9)}$ From the ratio of the respective constants, a $K(D)$ value of $514 \mathrm{~nm}$ was determined. The experiment was repeated in reverse orientation, with TPO immobilized to an aminosilane cuvette and differ-

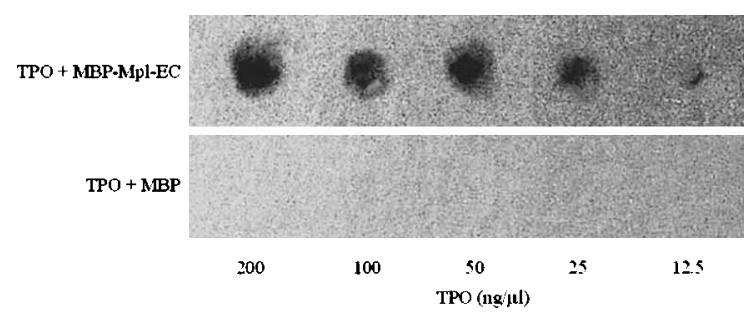

Fig. 3. Binding of Purified Mal-Mpl-EC to TPO in the Dot-Blot Assay

TPO $(5 \mu$ l) was spotted onto a PVDF membrane. The membrane was incubated with $0.5 \mu \mathrm{g} / \mathrm{ml}$ of MBP or MBP-Mpl-EC fusion protein overnight at $4{ }^{\circ} \mathrm{C}$. The bound protein was detected by alkaline phosphatase-conjugated goat anti-rabbit antibodies as described in Materials and Methods.



Fig. 4. Binding of TPO to Immobilized MBP-Mpl-EC

The interaction between MBP-Mpl-EC and TPO was monitored by surface plasmon resonance using an IAsys Affinity Biosensor. MBP-Mpl-EC was immobilized on the cuvette surface via NHS/EDC chemistry. To determine the $k_{\text {sss }}$ and $k_{\text {diss }}$ values of TPO for MBP-Mpl-EC, TPO was injected over immobilized MBP-Mpl-EC at concentrations ranging from 0.41 to $2.06 \mu \mathrm{M}$. ent concentrations of MBP-Mpl-EC were injected over the immobilized ligand, revealing similar $k_{\text {ass }}$ and $k_{\text {diss }}$ values of $6601.71 \mathrm{~m}^{-1} \mathrm{~s}^{-1}$ and $0.00324 \mathrm{~m}^{-1}$, and $K(D)=490.4 \mathrm{~nm}$ (data not shown). Furthermore, no nonspecific binding was evident when MBP was used as a negative control (data not shown).

In conclusion, the successful production of soluble human recombinant Mpl-EC, expressed in a prokaryotic system, has been achieved for the first time. The specificity of the binding was further demonstrated by the interaction between TPO and MBP-Mpl-EC or MBP using a dot-blot assay. The purified MBP does not bind any TPO protein, indicating that the interaction is mediated through the Mpl-EC of MBP-MplEC. The real-time sensorgram with a change in RU over time showed a strongly concentration-dependent binding of TPO to the surface-immobilized MBP-Mpl-EC. These observations are conclusive evidence that the MBP-Mpl-EC is correctly folded.

Acknowledgment This work was supported by China National Science Foundation Grant (39970410).

\section{REFERENCES}

1) Bartley T. D., Bogenberger J., Hunt P., Li Y. S., Lu H. S., Martin F., Chang M. S., Samal B., Nichol J. L., Swift S., Johnson M. J., Hsu R. Y., Parker V. P., Suggs S., Skrine J. D., Merewether L. A., Clogston C., Hsu E., Hokom M. M., Hornkohl A., Choi E., Pangelinan M., Sun Y., Mar V., McNinch J., Simonet L., Jacobsen F., Xie C., Shutter J., Chute H., Basu R., Selander L., Trollinger D., Sieu L., Padilla D., Trail G., Elliott G., Izumi R., Covey T., Crouse J., Garcia A., Xu W., del Castillo J., Biron J., Cole S., Hu M. C. T., Pacifici R., Ponting I., Saris C., Wen D., Yung Y. P., Lin H., Bosselmann R. A., Cell, 77, $1117-1124$ (1994).

2) de Sauvage F. J., Hass P. E., Spencer S. D., Malloy B. E., Gurney A. L., Spencer S. A., Darbonne W. C., Henzel W. J., Wong S. C., Kuang W. J., Oles K. J., Hultgren B., Solberg L. A., Jr., Goeddel D. V., Eaton D. L., Nature (London), 369, 533-538 (1994).

3) Lok S., Kaushansky K., Holly R. D., Kuijper J. L., Lofton-Day C. E., Oort P. J., Grant F. J., Heipel M. D., Burkhead S. K., Kramer J. M., Bell L. A., Sprecher C. A., Blumberg H., Johnson R., Prunkard D., Ching A. F. T., Mathewes S. L., Balley M. C., Forstrom J. W., Buddle M. M., Osborn S. G., Evans S. J., Sheppard P. O., Presnell S. R., O’Hara P. J., Hagen F. S., Roth G. J., Foster D. C., Nature (London), 369, 565-568 (1994).

4) Wendling F., Maraskovsky E., Debili N., Florindo C., Teepe M., Titeux M., Methia N., Breton-Gorius J., Cosman D., Vainchenker W., Nature (London), 369, 519-520 (1994).

5) Alexander W. S., Metcalf D., Dunn A. R., EMBO J., 15, 5569-5578 (1995).

6) Vigon I., Mornon J. P., Cocault L., Mitjavila M. T., Tambourin P., Gisselbrecht S., Souyri M., Proc. Natl. Acad. Sci. U.S.A., 15, 5640-5644 (1992).

7) Hsieh D. P., Huxtable S., Ng K. F., Chen H. M., Tsang P. W., Wang J., Xu P., Int. J. Biochem. Cell. Biol., 32, 481-488 (2000).

8) George A. J., French R. R., Glennie M. J., J. Immunol. Methods, 183, 51-63 (1995).

9) Gill A., Leatherbarrow R. J., Hoare M., Pollard-Knight D. V., Lowe P. A., Fortune D. H., J. Biotechnol., 48, 117-127 (1996). 